\title{
Molecular based temperature and strain rate dependent yield criterion for anisotropic elastomeric thin films
}

\author{
F. Bosi ${ }^{a, *}$, S. Pellegrino ${ }^{a}$ \\ ${ }^{a}$ Graduate Aerospace Laboratories, California Institute of Technology, Pasadena, CA, 91125, USA
}

\begin{abstract}
A molecular formulation of the onset of plasticity is proposed to assess temperature and strain rate effects in anisotropic semicrystalline rubbery films. The presented plane stress criterion is based on the strain rate-temperature superposition principle and the cooperative theory of yielding, where some parameters are assumed to be material constants, while others are considered to depend on specific modes of deformation. An orthotropic yield function is developed for a linear low density polyethylene thin film. Uniaxial and biaxial inflation experiments were carried out to determine the yield stress of the membrane via a strain recovery method. It is shown that the $3 \%$ offset method predicts the uniaxial elastoplastic transition with good accuracy. Both the tensile yield points along the two principal directions of the film and the biaxial yield stresses are found to obey the superposition principle. The proposed yield criterion is compared against experimental measurements, showing excellent agreement over a wide range of deformation rates and temperatures.
\end{abstract}

Keywords:

Yield stress, Cooperative model, Semi-crystalline polymers

\section{Introduction}

Polymer films are extensively used in engineering membrane systems such as stretchable electronics, soft robotics, inflatable civil and aerospace structures. Knowledge of the elastoviscoplastic limit is fundamental in the design of such systems, also accounting for their pronounced deformation rate and temperature dependence.

Semi-crystalline polymers often show an arrangement of radially aggregated crystalline lamellae and amorphous layers, resulting in an initial isotropic response [1]. When these polymers undergo large plastic deformation, anisotropy develops due to alignment of the lamellae and macromolecules chains of the amorphous phase, with the spherulitic structure reorganizing into a highly oriented solid characterized by alternating parallel amorphous and crystalline layers. Therefore, their macroscopic material response has been modeled as a two-phase composite through multi-scale approaches [2, 3]. The viscoplastic flow often starts in the amorphous region and then transitions to the crystalline zone [4]. The main deformation mechanism of the amorphous phase is interlamellar shear, while the viscoplastic deformation of the crystalline region consist of crystal twinning and slip [3].

Temperature and deformation rate effects on the yield stress have been extensively analyzed. Many molecular models have been established in isotropic plasticity to describe the yield stress of both amorphous and semi-crystalline polymers [5]. The

\footnotetext{
*Corresponding author

Email addresses: fbosi@caltech.edu (F. Bosi), sergiop@caltech.edu (S. Pellegrino)
}

Eyring theory has been widely applied to characterize yielding as a thermally activated process [6]. It assumes that irreversible deformations appear when the chain segments overcome an energy barrier. However, this single molecular process is not able to predict the elastic threshold over wide ranges of time and temperature. In order to overcome this limitation, the Ree-Eyring theory of viscosity was later developed; it assumes that two different rheological processes act in parallel [7]. The $\alpha$ and $\beta$ activation mechanisms have been employed for the characterization of the yield point of both amorphous [8] and semi-crystalline [9] polymers, below and above the glass transition temperature [10, 11]. More recently, many authors [12, 13, 14] have further extended the Eyring models through the formulation of the cooperative theory of yielding [15] and the strain rate-temperature superposition principle [16]. The latter model explains the macroscopic irreversible deformation as resulting from the mutual motion of several polymer chains over an energy barrier. Robertson proposed a molecular theory where the plastic flow is caused by the shear stress changing the structural arrangement of polymer chains [17]. Argon described the shear yielding of glassy polymers as the thermally activated formation of pairs of molecular kinks [18], while Bowden assumed that the yield process starts with the nucleation of small disc-shaped sheared regions [19].

Elastomeric films do not show a distinct yield point, which is usually associated with a load drop. Therefore, the experimental determination of the elasto-viscoplastic threshold strongly depends on the methodology employed for its detection and the choice of the minimal irreversible strain value [20]. In order to overcome the difficulty of a precise measurement of the yield stress, a strain recovery method has been suggested [21]. 
Several yield criteria have been suggested to quantify the dependence of the elastic loci on the hydrostatic component of stress in isotropic polymers [22]. Examples include the DruckerPrager model [23], the pressure-modified Tresca and Von Mises criteria [24, 25], and the Deshpande-Fleck yield criterion for foams [26]. All the aforementioned models assume material isotropy, whereas the effect of deformation rate and temperature on yield criteria for anisotropic polymers have been rarely addressed.

The aim of this study is to establish a yield criterion that accounts for the effects of temperature and deformation rate. The proposed approach combines the molecular theory of polymer plasticity with an anisotropic yield criterion for elastomeric membranes. In particular, the cooperative theory of yielding [27] is extended in order to consider the dependence of the yield point on the deformation modes. This model is implemented into a plane stress yield function based on Hill's criteria for anisotropic materials [28]. An application of the proposed formulation is presented considering orthotropic semi-crystalline polyethylene films that constitute the envelope of stratospheric balloons employed by the NASA Balloon Program.

The paper is organized as follows: Sect. 2 shows the analytical formulation of the molecular model for the yield stress of semi-crystalline polymers and its extension to anisotropic plasticity. The experimental procedures for the determination of the uniaxial and biaxial yield stresses are presented in Sect. 3 . while the results, identification of the model parameters and formulation of the yield criterion are presented in Sect. 4. Sect.5 concludes the paper.

\section{Modeling of yielding}

\subsection{Cooperative model of yield stress}

Although a single molecular process has been employed to assess the temperature and deformation rate dependence of the yield point for amorphous glassy polymers [16], it has been shown that a mechanism where the motion of several molecular chains contributes to yielding is more suitable for semicrystalline polymers [14]. This model is based on $n$ cooperative jumps of chains over a potential barrier and it was introduced by Fotheringham and Cherry for linear polyethylene [15]. The strain rate $\dot{\epsilon}$ at yield is then given by

$$
\dot{\epsilon}=\dot{\epsilon}^{*} \sinh ^{n}\left(\frac{\sigma_{\mathrm{eff}} V}{2 k T}\right),
$$

where $\dot{\epsilon}$ is the strain rate, $\dot{\epsilon}^{*}=\dot{\epsilon}^{*}(\Delta H, T)$ is a characteristic strain rate, which is function of an activation energy $\Delta H$ and temperature $T$ (in $\mathrm{K}$ ), $k$ is the Boltzmann's constant, and $V$ is an activation volume.

The activated rate process of plastic deformation is driven by the effective stress $\sigma_{\text {eff }}$ which is defined as [29]

$$
\sigma_{\mathrm{eff}}=\sigma_{y}-\sigma_{i}
$$

where $\sigma_{y}$ is the total stress applied to the system at yield, and $\sigma_{i}$ is a structural parameter associated with the recovery process from the past deformation history.
An expression for the yield stress can be obtained from Eqs. (1) and (2) as

$$
\frac{\sigma_{y}}{T}=\frac{\sigma_{i}}{T}+\frac{2 k}{V} \sinh ^{-1}\left(\frac{\dot{\epsilon}}{\dot{\epsilon}^{*}}\right)^{1 / n} .
$$

For thermo-rheologically simple materials, temperature effects are represented by a shift in the time (or frequency) scale, as postulated by the time-temperature superposition principle (TTSP). In a similar vein, the effect of high (low) temperature on the yield point of a polymer can be associated with the response produced at low (high) deformation rates through the so called strain rate-temperature superposition principle [16]. As a result, the isothermal curves that define the reduced yield stress $\sigma_{y} / T$ as a function of the logarithm of the deformation rate $\log \dot{\epsilon}$ can be moved in order to obtain a master curve for a given reference temperature $T_{\text {ref }}$. The reduced yield stress master curve is created by introducing both a vertical shift, $\Delta\left(\sigma_{y} / T\right)$, and a horizontal shift, $\Delta(\log \dot{\epsilon})$, defined as [30, 27]

$$
\begin{aligned}
\Delta\left(\frac{\sigma_{y}}{T}\right) & =\frac{\sigma_{y}\left(T_{\text {ref }}\right)}{T_{\text {ref }}}-\frac{\sigma_{y}(T)}{T}, \\
\Delta(\log \dot{\epsilon}) & =\log \dot{\epsilon}\left(T_{\text {ref }}\right)-\log \dot{\epsilon}(T) .
\end{aligned}
$$

Although in some studies the master curve has been built only by means of a horizontal shift [13, 31], the combination of lateral and vertical shifts often allows a better representation of the experimental measurements [12, 30].

Inserting the cooperative model definition of the yield stress, Eq. (3), into Eq. (4a), yields

$$
\begin{aligned}
\Delta\left(\frac{\sigma_{y}}{T}\right)= & \frac{\sigma_{i}\left(T_{\text {ref }}\right)}{T_{\text {ref }}}-\frac{\sigma_{y}(T)}{T} \\
& +\frac{2 k}{V}\left(\sinh ^{-1}\left(\frac{\epsilon\left(\dot{T}_{\text {ref }}\right)}{\dot{\epsilon}^{*}\left(T_{\text {ref }}\right)}\right)^{1 / n}-\sinh ^{-1}\left(\frac{\epsilon(T)}{\dot{\epsilon}^{*}(T)}\right)^{1 / n}\right) .
\end{aligned}
$$

Since the vertical shift does not depend on the deformation rate [16], the last term of Eq. (5) should vanish, leading to the relation

$$
\frac{\dot{\epsilon}\left(T_{\mathrm{ref}}\right)}{\dot{\epsilon}(T)}=\frac{\dot{\epsilon}^{*}\left(T_{\mathrm{ref}}\right)}{\dot{\epsilon}^{*}(T)},
$$

which can be inserted into Eq. (4b) in order to express the vertical and horizontal transformations as a function of the parameters $\sigma_{i}$ and $\dot{\epsilon}^{*}$

$$
\begin{aligned}
\Delta\left(\frac{\sigma_{y}}{T}\right) & =\frac{\sigma_{i}\left(T_{\text {ref }}\right)}{T_{\text {ref }}}-\frac{\sigma_{i}(T)}{T}, \\
\Delta(\log \dot{\epsilon}) & =\log \dot{\epsilon}^{*}\left(T_{\text {ref }}\right)-\log \dot{\epsilon}^{*}(T) .
\end{aligned}
$$

For semi-crystalline polymers, the shifts in Eq. (7) depend on the temperature through a linearized form of Arrhenius law [16, 32]

$$
\begin{gathered}
\Delta\left(\frac{\sigma_{y}}{T}\right)=A\left(\frac{1}{T}-\frac{1}{T_{\text {ref }}}\right), \\
\Delta(\log \dot{\epsilon})=B\left(\frac{1}{T}-\frac{1}{T_{\text {ref }}}\right),
\end{gathered}
$$


where $A$ and $B$ are material constants. By comparing the right hand side of Eqs. (7a) and (8a) we obtain

$$
\sigma_{i}(T)=\sigma_{i}(0)-m T,
$$

where $\sigma_{i}(0)=-A$ is the internal stress at the absolute temperature, and $m=\left(\sigma_{i}(0)-\sigma_{i}\left(T_{\text {ref }}\right)\right) / T_{\text {ref }}$ is a constant independent of the choice of $T_{\text {ref. }}$. In a similar vein, equating relations (7b) and $(8 \mathrm{~b})$ and changing the base of the logarithm yields

$$
\dot{\epsilon}^{*}(T)=\dot{\epsilon}_{0} \exp \left(-\frac{\Delta H}{k T}\right),
$$

where $\Delta H=B k \ln 10$ is the activation energy, while $\dot{\epsilon}_{0}$ is a pre-exponential factor

$$
\dot{\epsilon}_{0}=\dot{\epsilon}^{*}\left(T_{\text {ref }}\right) \exp \left(\frac{\Delta H}{k T_{\text {ref }}}\right) .
$$

The formulation for the cooperative model of yielding is obtained by substituting Eqs. (9) and (10) into Eq. (3),

$$
\sigma_{y}=\sigma_{i}(0)-m T+\frac{2 k T}{V} \sinh ^{-1}\left(\frac{\dot{\epsilon}}{\dot{\epsilon}_{0} \exp \left(-\frac{\Delta H}{k T}\right)}\right)^{1 / n} .
$$

Equation (12) is a function that describes the yield stress of an isotropic semi-crystalline polymers by means of six parameters. The activation volume $V$ and activation energy $\Delta H$ can be seen as homogenized effective quantities that take into account both the amorphous and crystalline phases of the material [14].

\subsection{Anisotropic yield function}

The development of a yield criterion requires that the cooperative formulation for the yield stress, originally developed for isotropic plasticity, is extended to consider material anisotropy. The particular distribution of macromolecules and morphology of elastomers cause the dependence of the yield point on the deformation direction. Anisotropy is taken into account by assuming that the yield stresses in different material directions are described through the cooperative model, Eq. (12), where some parameters depend on the specific deformation modes.

We assume that the activation energy $\Delta H$, the activation volume $V$ and the pre-exponential factor $\dot{\epsilon}_{0}$ are material parameters independent of the mechanical loading conditions to which the polymer is subjected. It follows that the remaining constants, namely the athermal stress $\sigma_{i}(0)$ and the factors $m$ and $n$, are responsible for the loading direction dependence of the yield point. In fact, $\sigma_{i}(0)$ and $m$ describe the temperature variation of the initial internal stress $\sigma_{i}$ in the polymer, and hence rely upon the manufacturing process of the material and its morphology, which leads to different responses under various loading conditions. The parameter $n$ describes the mutual movement of chain segments responsible for plastic flow, therefore the yield event involves a different number of such transitions depending on the mono or multiaxial loading condition, with a higher $n$ associated with material deformation under biaxial stresses.

The aforementioned assumption leads to different yield stresses which describe the anisotropic response of the material, which are employed in the development of yield locus formulation.

In plasticity theory, the yield criterion is a function that describes the critical values of stress at which inelastic deformations develop. It is usually dependent on the six independent components of stress and its general form is

$$
f\left(\sigma_{11}, \sigma_{12}, \sigma_{13}, \sigma_{22}, \sigma_{23}, \sigma_{33}\right)=\text { constant. }
$$

This formulation can be further specified on the basis of physical conditions, therefore in the case of anisotropic thin films it has to take into account plane stress conditions, temperature and deformation rate dependence of the yield stress. An extension of the $J 2$ plasticity theory will be adopted in the development of the yield surface [33]. The generalized Hill's criterion is usually employed for anisotropic materials which possess three orthogonal symmetry planes, such as metals, polymers, and certain composites. In terms of principal material directions I and II, and for plane stress conditions it has the expression

$$
C \sigma_{\mathrm{I}}^{2}+D \sigma_{\mathrm{II}}^{2}+E \sigma_{\mathrm{I}} \sigma_{\mathrm{II}}=1,
$$

where $C=C(T, \dot{\epsilon}), D=D(T, \dot{\epsilon}), E=E(T, \dot{\epsilon})$ are material functions characteristic of the current state of anisotropy. This criterion can be adopted for orthotropic materials, so that the parameters in Eq. (14) are related to the basic yield stresses of the membrane. For uniaxial loading conditions along I and II, Eq. (14) yields

$$
C=\frac{1}{\sigma_{y, \mathrm{I}}^{2}}, \quad D=\frac{1}{\sigma_{y, \mathrm{II}}^{2}},
$$

where $\sigma_{y, \mathrm{I}}=\sigma_{y, \mathrm{I}}(T, \dot{\epsilon})$ and $\sigma_{y, \mathrm{II}}=\sigma_{y, \mathrm{II}}(T, \dot{\epsilon})$ are the temperature and strain rate dependent yield stresses along the two directions of orthotropy, defined by means of the cooperative formulation, Eq. (12). Finally, if the equibiaxial yield point $\sigma_{y, b}=\sigma_{y, b}(T, \dot{\epsilon})$ is known, the remaining parameter $E$ can be obtained as

$$
E=\frac{1}{\sigma_{y, b}^{2}}-\frac{1}{\sigma_{y, \mathrm{I}}^{2}}-\frac{1}{\sigma_{y, \mathrm{II}}^{2}} .
$$

\section{Experimental procedure}

\subsection{Material}

The film used to build the envelope of the NASA Superpressure balloons was selected for the present study [34]. This membrane is produced as a three layer extrusion of Linear Low Density Polyethylene (LLDPE), and is designated as StratoFilm 420. The total thickness of the film is $38 \mu \mathrm{m}$, with a thickness distribution of $20 / 60 / 20 \%$. The extrusion process consists of pulling the resin from a circular die along the machine direction (MD) and simultaneously stretching it in the transverse direction (TD). The biaxial elongation of the film is necessary to achieve nearly balanced properties between the two directions, although an anisotropic behavior is shown, especially under finite deformation. The glass transition temperature of this material is ca. $-95^{\circ} \mathrm{C}$, therefore LLDPE is in a rubbery state [35, 36].

A large strain orthotropic thermomechanical constitutive model has been developed to describe the viscoelastic behavior of the material over a wide range of temperatures, strain rates, and mechanical loading conditions [37, 38]. 


\subsection{Experimental setup}

The yield point of polymers is strongly affected by temperature and strain rate effects, therefore experiments were carried out at different thermal conditions and deformation rates. These were chosen in order to cover the range of temperatures and deformation rates experienced during a balloon flight, namely $T=$ $[+24 ;+10 ;-10 ;-30 ;-50]^{\circ} \mathrm{C}$ and $\dot{\epsilon}=[1 ; 0.1 ; 0.01 ; 0.001] \% \mathrm{~s}^{-1}$.

Tests were conducted with an Instron 3119-506 environmental chamber mounted on an Instron 5569 testing machine, equipped with an Instron 2525-816 load cell (R.C. 500N). Two type$\mathrm{K}$ thermocouples were located close to the specimen and the temperature was acquired through an Omega HH802U thermocouple reader interfaced with an Omega HH800SW software, showing oscillations within $\pm 0.3^{\circ} \mathrm{C}$ once a constant temperature had been reached. The VIC $3 \mathrm{D}^{\mathrm{TM}}$ (Correlated Solutions, v.7) three-dimensional Digital Image Correlation system was employed to measure displacements and strains [39]. Two stereomounted digital cameras Grasshopper 5.0 MP Mono FireWire 1394b (PointGrey) equipped with Schneider Kreuznach Xenoplan lenses (Focal length $35 \mathrm{~mm}$, F-number 8), and the VICSnap8 software (v.8) were used to acquire images. The cameras set-up and samples preparation were determined in order to minimize uncertainties and noise level during image correlation [40]. Black paint was lightly sprayed on the transparent samples in order to obtain a random speckle pattern, with average speckle dimension of 7 pixels, to avoid aliasing. The paint deposited on the sample surface did not modify the material response. In order to reduce displacement variance during postprocessing, an adequate image contrast was provided by positioning white LED lights underneath the specimen. The calibration of the stereo system was performed by acquiring more than 25 static images per camera (calibration target $12 \times 9$ dots, spacing $5 \mathrm{~mm}$ ), to achieve a calibration score lower than 0.03 pixels [41].

Uniaxial tension tests were carried out on dumb-bell shaped samples (cut with die A, ASTM D412) oriented along both MD and TD in order to assess any orthotropy of the elastic limit of the material. The bottom end of the specimen was held fixed, whereas the top end was attached to the crossbeam of the Instron machine, which was moved at a rate that matched the nominal strain rate of the experiment. At the beginning of each test, the sample was lightly pretensioned with a force of $0.05 \mathrm{~N}$ in order to remove any initial slackness. The measured load was divided by the initial cross-section area of the specimen $(12.9 \mathrm{~mm} \times$ $0.038 \mathrm{~mm}$ ) in order to compute the engineering stress. During image postprocessing, a correlation subset of $31 \times 31$ pixels, a step size of 7 pixels and a filter size of 13 pixels were chosen to achieve enough spatial resolution. The projection error during image correlation was always below 0.09 pixels, showing a very good correlation despite the presence of the thermal chamber window between the cameras and the specimen.

Diaphragm inflation experiments were carried out to characterize the yield stress of LLDPE under biaxial tension. During these tests, a $100 \mathrm{~mm}$ diameter membrane was clamped to a custom designed test box and inflated with air. The specimen was fixed to the test box by applying a small pre-tension (maximum pre-strain was lower than $0.1 \%$ ) in order to guarantee a flat initial
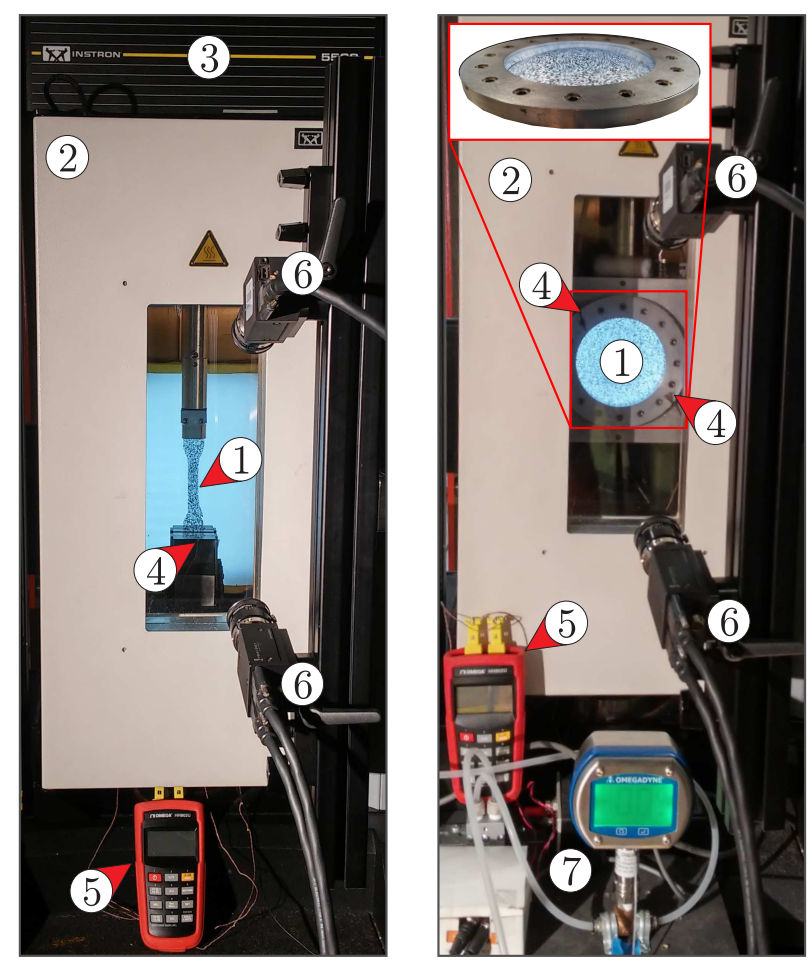

Figure 1: Experimental setup for uniaxial (left) and biaxial (right) tests on LLDPE specimens (1) placed inside a thermal chamber (2) mounted on an Instron testing machine (3). Thermocouples (4), thermocouple reader (5) and stereo-cameras (6) were used to acquire temperatures and images during tests. A custom pressure regulator and gauge were employed during inflation experiments (7).

surface, without wrinkles. For experiments performed at subambient temperatures, the sample was cooled for $30 \mathrm{~min}$ to reach the desired temperature, and then inflated. The pressure signal was generated through LabView SignalExpress (v.2.5.1) and the National Instruments (NI) USB-6221 data acquisition module (DAQ), connected to an analog device AD694JN voltage-tocurrent converter and an Omega IP610-030 pressure controller. An Omega DPG409-015G pressure gauge was interfaced with the DAQ in order to measure the inlet pressure. The pressure signal was calibrated for each test such that the engineering strain rate at the apex of the inflated membrane, where plasticity first develops, was always within $\pm 15 \%$ with respect to its desired value. For image correlation, a subset dimension of $33 \times 33$ pixels, step size of 7 pixels, and filter size of 13 pixels were chosen.

The experimental setups for both uniaxial and biaxial tests are shown in Fig. 1 .

\subsection{Yield stress determination}

The yield point of a material is usually associated with the peak in the stress-strain curve, where the onset of plastic flow becomes evident. However, elastomers soften without showing a decrease in the load carrying capacity, even at very large deformations when part of the strain is not recoverable anymore. Therefore, for materials in the rubbery state, several methods 


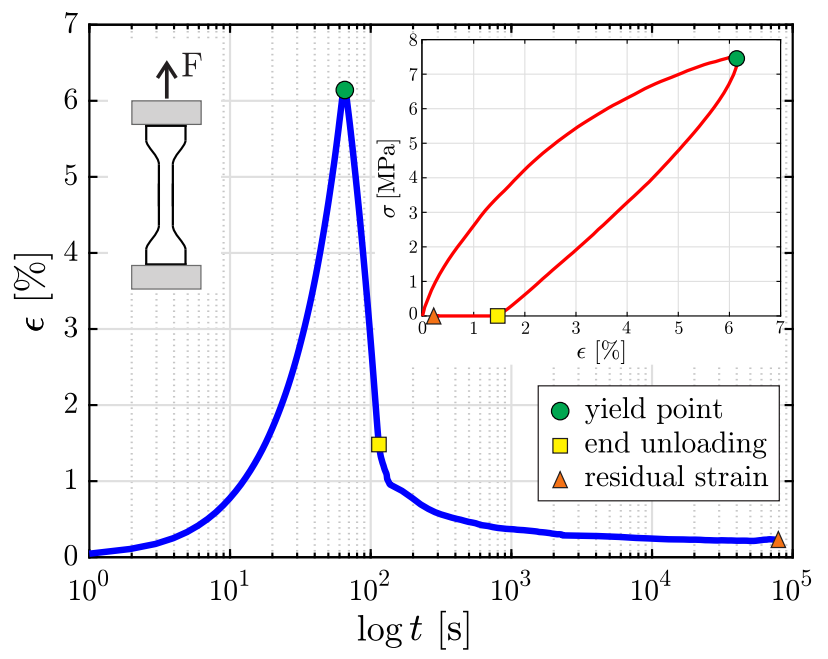

Figure 2: Time evolution of strain along extrusion direction (MD) for uniaxial tension test performed at $T=+24^{\circ} \mathrm{C}$ and $\dot{\epsilon}=0.1 \% \mathrm{~s}^{-1}$. The end of loading, unloading and unstressed strain recovery phases are marked with a dot, square and triangle. The residual strain after 24 hours is $\epsilon_{r}=0.19 \%$, therefore the green dot represents the yield point accordingly to the proposed yield stress determination procedure. The inset plot shows the corresponding stress-strain relation, where material non-linearity and hysteresis can be observed.

have been proposed in the literature to calculate the elastoplastic limit. The Considère geometric construction identifies the extrinsic yield point in a true stress-nominal strain plot [42]. The offset method is widely adopted, even though the amount of shift on the strain axis is an arbitrary choice and is usually chosen between $1 \%$ [13] and 2\% [43]. If accuracy is needed in the determination of the onset of plasticity, a true yield stress procedure via the strain recovery method is desirable.

The true yield stress procedure will be employed in the present study. Once the temperature and deformation rate of the tests have been selected, several specimens are deformed up to different nominal strains that produce small unrecoverable deformations. After unloading and strain recovery, the residual deformation $\epsilon_{r}$ is measured. Hence, each strain level $\epsilon$ corresponds to a residual deformation $\epsilon_{r}$, which is plotted as a function of the applied strain $\epsilon$ and it is back-extrapolated in order to find the strain level that produces zero plastic deformation, which is defined as the yield strain $\epsilon_{y}$. The yield point is identified as the stress corresponding to $\epsilon_{y}$ on the stress-strain curve [44, 45]. However, an accurate analysis of very small inelastic strains proved that there is no evidence of a strain threshold below which the residual deformation disappears entirely [25]. Since DIC measurements provide the strain field throughout the whole specimen, our experiments confirmed that small non-zero unrecoverable strains are present even for relatively small initial deformations. For this reason, instead of using the back-extrapolation to zero residual strain, in the following the plastic onset point is defined as the biggest stress that produces an irreversible deformation $\epsilon_{r} \leq 0.2 \%$. This choice is a reasonable engineering assumption for materials capable of undergoing large deformations without showing any significant residual strain. Indeed, the uniaxial mechanical yield strains $\epsilon_{y}$ were found to be always higher than $5 \%$

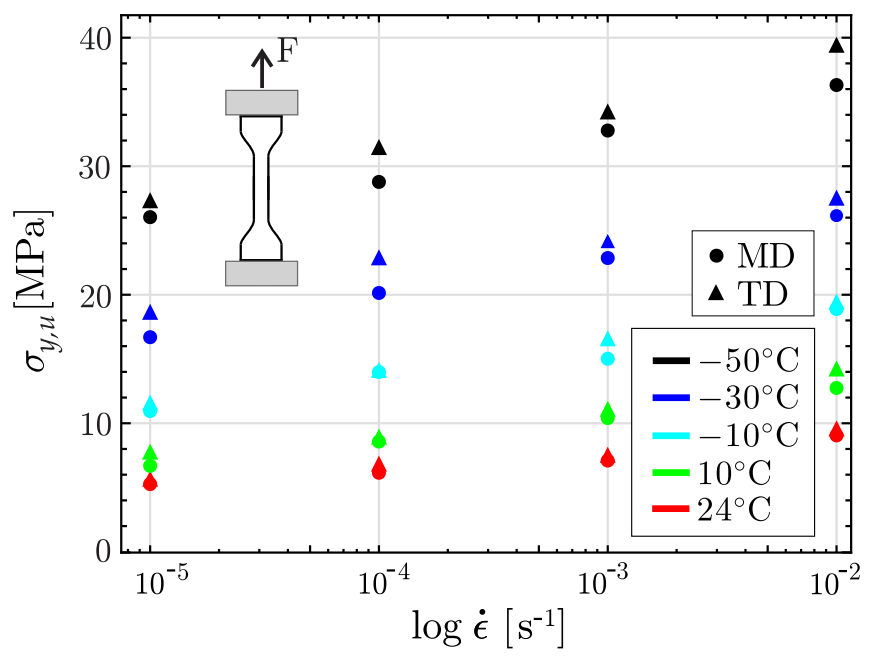

Figure 3: Uniaxial yield stresses $\sigma_{y, u}$ along MD (dot) and TD (triangle) directions for $T=[+24 ;+10 ;-10 ;-30 ;-50]^{\circ} \mathrm{C}$ and $\dot{\epsilon}=[1 ; 0.1 ; 0.01 ; 0.001] \% \mathrm{~s}^{-1}$.

for all the temperatures and strain rates considered (see Figs. 2 and 4).

For all temperatures considered, the residual strain has been evaluated between the initial and final unstressed state, at room temperature $\left(+24^{\circ} \mathrm{C}\right)$. Therefore, for both uniaxial and biaxial experiments performed at sub-ambient temperatures, the samples had to be cooled, then loaded and unloaded, and finally brought back to ambient temperature for strain recovery. The amount of residual deformation, which is strongly dependent on viscoelastic effects, was measured after 12 hours from the end of each test. If the resulting inelastic strain $\epsilon_{r}$ was considerably lower (higher) than $0.2 \%$, the following test was carried out at a higher (lower) deformation, increasing (decreasing) the final displacement of the tensile machine by $2 \mathrm{~mm}$ or the inlet pressure by $250 \mathrm{~Pa}$, for uniaxial and biaxial tests respectively. Once the yield point had been identified through this incremental method, two other experiments were performed for the same amount of final displacement or maximum pressure, with $13 \%$ as the largest variability. In summary, an average of 6 tests was necessary to detect the yield point for each combination of temperature, strain rate, and loading condition, therefore ca. 350 experiments were performed in the present study.

Figure 2 presents an application of the procedure to a uniaxial tension test along MD performed at $T=+24^{\circ} \mathrm{C}$ and $\dot{\epsilon}=0.1 \% \mathrm{~s}^{-1}$, where the yield point has been hit since the residual strain was $0.19 \%$. As shown in the plot, the selected recovery time guarantees more than $99 \%$ of recovered strain with respect to 24 hours. The zero-tangent of the residual deformation curve after one day from the end of the test corresponds to the amount of irreversible strain present in the specimen.

\section{Results and discussion}

\subsection{Uniaxial yield stresses}

An accurate plane stress yield criterion for orthotropic polyethylene membranes requires the determination of at least 


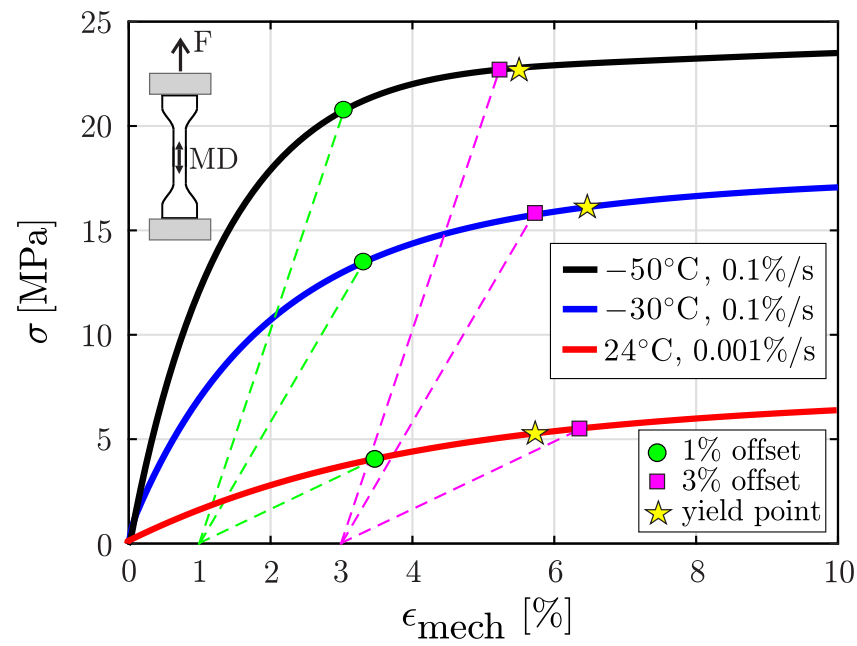

Figure 4: Comparison between the 1\% (circle) and 3\% (square) offset method predictions and the yield point (star) detected via strain recovery method for uniaxial tension experiment along the extrusion direction (MD) performed at $T=[+24 ;-10 ;-30]^{\circ} \mathrm{C}$, and $\dot{\epsilon}=[0.1 ; 0.001] \% \mathrm{~s}^{-1}$.

three yield stresses for each temperature and strain rate considered, as described in Sect. 3.2. Therefore uniaxial tension tests along the two principal directions and biaxial inflation experiments were performed in order to identify the yield points by means of the proposed strain recovery method.

The results of the uniaxial experiments are shown in Fig. 3. where the yield point of the material is reported as a function of the deformation rate for the temperatures considered. The yield limit is higher at lower temperatures and faster strain rates, confirming the well-known behavior of polymers. Because the transverse direction (TD) is stiffer than the machine direction (MD), it can be observed that the yield stress along TD is always higher than along MD.

The true yield stress $\sigma_{y, u}$ in Fig. 3 has been compared with the prediction of the elastoplastic limit obtained by means of the traditional methods, namely the Considère construction and the offset method. The former geometric method is not applicable since for the majority of the experiments there is no tangent to the stress-strain curve through the abscissa $\epsilon=-1$ for $\epsilon<20 \%$. The offset yield point is obtained through the intersection between the stress-strain curve and a horizontally shifted line drawn at a slope equal to the initial Young's modulus, which is defined as the secant between the unstressed state and $\epsilon=1 \%$. In Fig. 4 the $1 \%$ and $3 \%$ offset predictions are reported together with the yield point detected via the strain recovery method for three different temperatures $\left(+24^{\circ} \mathrm{C},-10^{\circ} \mathrm{C}\right.$, and $\left.-30^{\circ} \mathrm{C}\right)$ and two deformation rates $\left(0.1 \% \mathrm{~s}^{-1}\right.$ and $\left.0.001 \% \mathrm{~s}^{-1}\right)$. It can be noticed that the $3 \%$ offset method gives good predictions of the true yield stress of the material, while the $1 \%$ and $2 \%$ offsets underestimate the elastoplastic transition. This analysis has been conducted over the full set of experiments, and it has been concluded that the $3 \%$ offset is able to predict the yield stress with errors smaller than $\pm 8 \%$ over the full range of temperatures and deformation rates investigated.

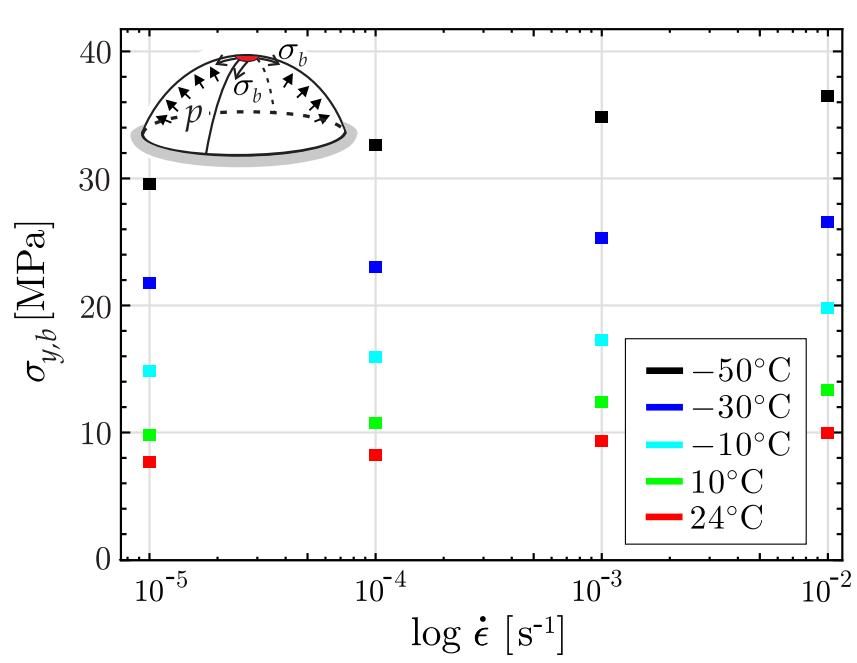

Figure 5: Equibiaxial yield stresses $\sigma_{y, b}$ of LLDPE thin membrane for $T=$ $[+24 ;+10 ;-10 ;-30 ;-50]^{\circ} \mathrm{C}$ and $\dot{\epsilon}=[1 ; 0.1 ; 0.01 ; 0.001] \% \mathrm{~s}^{-1}$.

\subsection{Biaxial yield stresses}

During the biaxial tests, strain localizes near the apex of the inflated film. The stresses in this region are higher because the local curvatures of the membrane are smaller. The nominal yield stress $\sigma_{y, b}$ was assumed to be isotropic and hence given by

$$
\sigma_{y, b}=\frac{p \bar{r}}{2 t}
$$

where $p$ is the inlet pressure, $\bar{r}$ is the mean radius of curvature between the two principal directions MD and TD, and $t$ is the initial thickness of the membrane $(38 \mu \mathrm{m})$. Since the orthotropy of StratoFilm 420 is not very pronounced [35], with a difference of ca. $12 \%$ between the two directions, the isotropic assumption has been shown to correctly predict the average stress at the center of the thin circular membrane [38]. Through this assumption, the yield point obtained by means of diaphragm inflation experiments represents an equi-biaxial limit as reported in Fig. 5 ,

Thermal and deformation rate effects, which have already been shown to strongly affect the uniaxial behavior, also significantly influence the biaxial limit. There is an increase of the yield stress with an increase of the deformation rate or a decrease in temperature. However, it can be observed that the equibiaxial strengths are slightly higher than the uniaxial ones for lower strain rates and higher temperatures, while they approach the monoaxial values for high deformation rates and lower temperatures. This feature will lead towards a distorted locus along the equibiaxial stress state in the former case, while a Von Mises-like domain will be defined in the latter case.

\subsection{Identification of model parameters}

Figures 3 and 5 indicate that both the uniaxial yield points along the two principal directions and the equibiaxial yield points obey the strain rate-temperature superposition principle. Therefore, three different experimental master curves can be built, one for each direction of uniaxial loading and a third one 


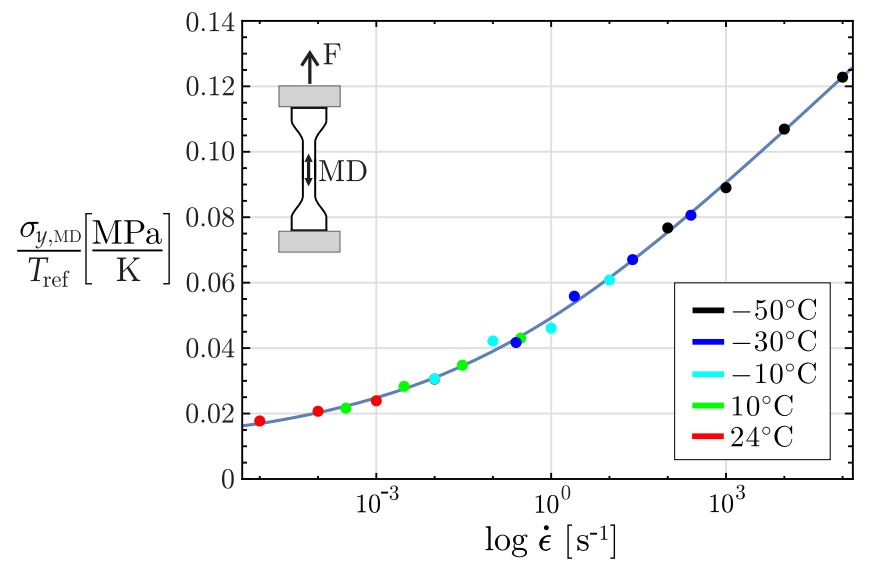

Figure 6: Reduced yield stress master curve at $T_{\text {ref }}=24^{\circ} \mathrm{C}$ for LLDPE thin film tested under uniaxial tension along MD.

for biaxial loading, and each of these curves may be expressed by means of the cooperative model, Eq. (12). However, this approach would require the determination of eighteen independent constants, six per each loading conditions, while some of the parameters, namely $\left[\Delta H, V, \dot{\epsilon}_{0}\right]$, have been assumed as material constants, Sect.2.2 Considering this assumption, three master curves were obtained, each characterized by a set of parameters $\left[\sigma_{i}(0), m, n\right]$ that define the anisotropic behavior of the polyethylene film.

Starting from the yield stress along MD, the first six parameters $\left(\sigma_{i, \mathrm{MD}}(0), m_{\mathrm{MD}}, V, \Delta H, \dot{\epsilon}_{0}, n_{\mathrm{MD}}\right)$ were obtained through the following procedure [27]. The first step consisted in constructing a reduced yield stress master curve for MD at a reference temperature $T_{\text {ref }}=+24^{\circ} \mathrm{C}$. The experimentally obtained yield stresses along MD at $T \neq T_{\text {ref }}$, shown in Figs. 3, were horizontally and vertically shifted in an Eyring plot $\left(\sigma_{y} / T_{\text {ref }}\right.$ vs. $\left.\log \dot{\epsilon}\right)$ in order to define the master curve for the chosen reference temperature. Once this curve had been obtained, the experimental data were fitted (least-squares FindFit in Mathematica v.11.0) by means of the cooperative definition of the yield stress, Eq. (3), evaluated at $T_{\text {ref }}$ and along MD

$$
\frac{\sigma_{y, \mathrm{MD}}}{T_{\text {ref }}}=\frac{\sigma_{i, \mathrm{MD}}\left(T_{\mathrm{ref}}\right)}{T_{\text {ref }}}+\frac{2 k T}{V} \sinh ^{-1}\left(\frac{\dot{\epsilon}}{\dot{\epsilon}_{0}^{*}\left(T_{\text {ref }}\right)}\right)^{1 / n_{\mathrm{MD}}},
$$

in order to obtain the parameters $\left[\sigma_{i, \mathrm{MD}}\left(T_{\text {ref }}\right), V, \dot{\epsilon}_{0}^{*}\left(T_{\text {ref }}\right), n_{\mathrm{MD}}\right]$. The remaining constants were determined from the horizontal and vertical shifts, Eqs. (8)-(11), which were employed in the construction of the master curve. In particular, from the temperature dependence of the vertical shifts used in the determination of the reduced yield stress master curve, $\sigma_{i, \mathrm{MD}}(0)$ was obtained through Eq. 8a), and hence the parameter $m_{\mathrm{MD}}$ was calculated as

$$
m_{\mathrm{MD}}=\frac{\sigma_{i, \mathrm{MD}}(0)-\sigma_{i, \mathrm{MD}}\left(T_{\mathrm{ref}}\right)}{T_{\mathrm{ref}}} .
$$

In a similar vein, from the analysis of the horizontal shifts at different temperatures, the activation energy $\Delta H$ was computed from Eq. (8b). Finally, the pre-exponential factor $\dot{\epsilon}_{0}$ was obtained by means of Eq. (11).

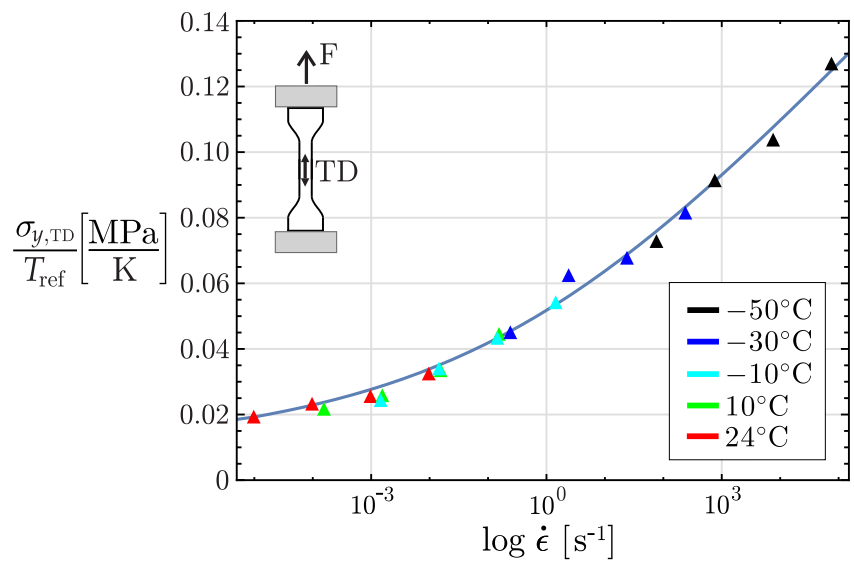

Figure 7: Reduced yield stress master curve at $T_{\text {ref }}=24^{\circ} \mathrm{C}$ for LLDPE thin film tested under uniaxial tension along TD.

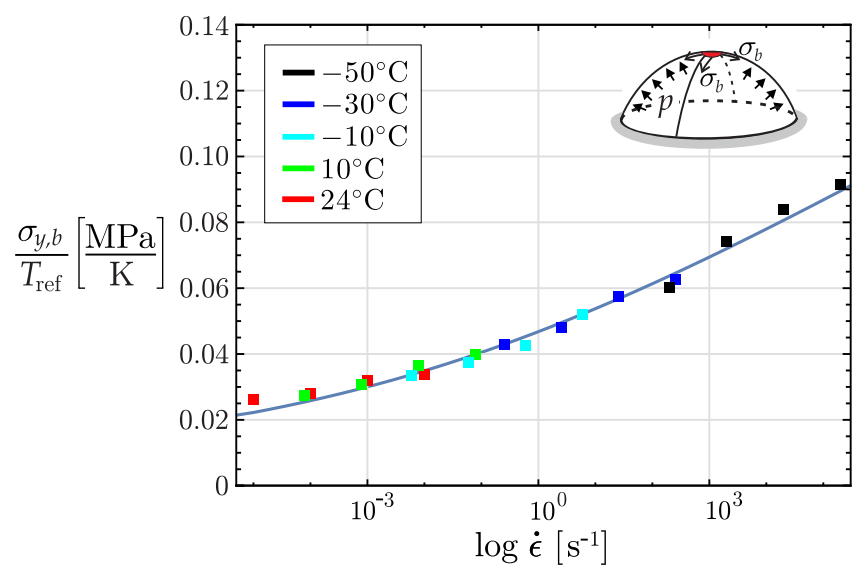

Figure 8: Reduced yield stress master curve at $T_{\text {ref }}=24^{\circ} \mathrm{C}$ for LLDPE thin film tested under equibiaxial tension through diaphragm inflation experiments.

Once the material constants $\left[\Delta H, V, \dot{\epsilon}_{0}\right]$ had been fixed, the remaining parameters were calculated independently for both the uniaxial TD and the equibiaxial yield points following the aforementioned procedure. Note that, keeping fixed the preexponential factor $\dot{\epsilon}_{0}$ and the activation energy $\Delta H$ implies that the horizontal shifts employed in the construction of the master curve are common to all loading conditions, for the same thermal status. It has to be observed that the material parameters $\Delta H$ and $V$ are assumed to be independent of temperature and deformation rate, whereas they depend on the volume fraction of the crystalline region [14].

The master curves developed through the strain ratetemperature superposition principle can predict the yield stresses of the material for deformation rates and temperatures different from those analyzed in this study. In particular, the elastoplastic transition at dynamic strain rates can be extrapolated without carrying out further experiments, as shown in Figs. 6, 7 and 8 for uniaxial tension along the two principal directions MD and $\mathrm{TD}$, and under equibiaxial tension. The yield points for all three loading conditions are very well captured by cooperative models with equal parameters $\left[\Delta H, V, \dot{\epsilon}_{0}\right]$ and different parameters 
Table 1: Cooperative model parameters

\begin{tabular}{llll}
\hline Parameters & Uniaxial MD & Uniaxial TD & Equibiaxial \\
\hline$\sigma_{i}(0)[\mathrm{MPa}]$ & 34.6165 & 42.7274 & 61.7206 \\
$m[\mathrm{MPa} / \mathrm{K}]$ & 0.1081 & 0.1331 & 0.2029 \\
$n$ & 6.9127 & 6.9127 & 11.4659 \\
\hline$V\left[\mathrm{~m}^{3}\right]$ & & $4.4541 \cdot 10^{-28}$ & \\
$\Delta H[\mathrm{~J} / \mathrm{mol}]$ & & $1.9901 \cdot 10^{-19}$ & \\
$\dot{\epsilon}_{0}\left[\mathrm{~s}^{-1}\right]$ & & $2.4669 \cdot 10^{21}$ & \\
\hline
\end{tabular}

$\left[\sigma_{i}(0), m, n\right]$. They can be written as

$$
\begin{aligned}
& \sigma_{y, \mathrm{MD}}=\sigma_{i, \mathrm{MD}}(0)-m_{\mathrm{MD}} T+\frac{2 k T}{V} \sinh ^{-1}\left(\frac{\dot{\epsilon}}{\dot{\epsilon}_{0} \exp \left(-\frac{\Delta H}{k T}\right)}\right)^{1 / n_{\mathrm{MD}}}, \\
& \sigma_{y, \mathrm{TD}}=\sigma_{i, \mathrm{TD}}(0)-m_{\mathrm{TD}} T+\frac{2 k T}{V} \sinh ^{-1}\left(\frac{\dot{\epsilon}}{\dot{\epsilon}_{0} \exp \left(-\frac{\Delta H}{k T}\right)}\right)^{1 / n_{\mathrm{TD}}}, \\
& \sigma_{y, b}=\sigma_{i, b}(0)-m_{b} T+\frac{2 k T}{V} \sinh ^{-1}\left(\frac{\dot{\epsilon}}{\dot{\epsilon}_{0} \exp \left(-\frac{\Delta H}{k T}\right)}\right)^{1 / n_{b}} .
\end{aligned}
$$

The constants defining the yield models in Eq. (20) are reported in Table 1 . It can be noticed that the number of polymer chains $n$ involved in the yield process is equal if the material is subjected to uniaxial tension along one of the two principal directions, while it is noticeably higher when the deformation mechanism is multiaxial. This result shows that the biaxial yield process requires the cooperative movement of more polymer chains oriented in different directions.

\subsection{Yield criterion}

The experimental investigation of the temperature and strain rate dependence of the yield limits in Sect. 3 and 4.3 has been focused on the principal material directions MD and TD. The orthotropic plane stress yield function proposed by Hill [33] and presented in Sect. 2.2 can be specified for LLDPE in terms of principal stresses $\sigma_{\mathrm{MD}}$ and $\sigma_{\mathrm{TD}}$. Hence, Eq. (14) can be rewritten as

$$
C \sigma_{\mathrm{MD}}^{2}+D \sigma_{\mathrm{TD}}^{2}+E \sigma_{\mathrm{MD}} \sigma_{\mathrm{TD}}=1
$$

where $C, D$ and $E$ are experimentally derived functions, shown in Eqs. (15) and (16), that describe the temperature and deformation rate dependence of the yield stresses of the polymeric thin film. They can be specialized considering the direction of material orthotropy MD and TD

$$
\begin{aligned}
& C=\frac{1}{\sigma_{y, \mathrm{MD}}^{2}}, \quad D=\frac{1}{\sigma_{y, \mathrm{TD}}^{2}}, \\
& E=\frac{1}{\sigma_{y, b}^{2}}-\frac{1}{\sigma_{y, \mathrm{MD}}^{2}}-\frac{1}{\sigma_{y, \mathrm{TD}}^{2}},
\end{aligned}
$$

where the uniaxial and equibiaxial yield stresses for the orthotropic polyethylene membrane are defined by means of the cooperative models in Eq. (20).

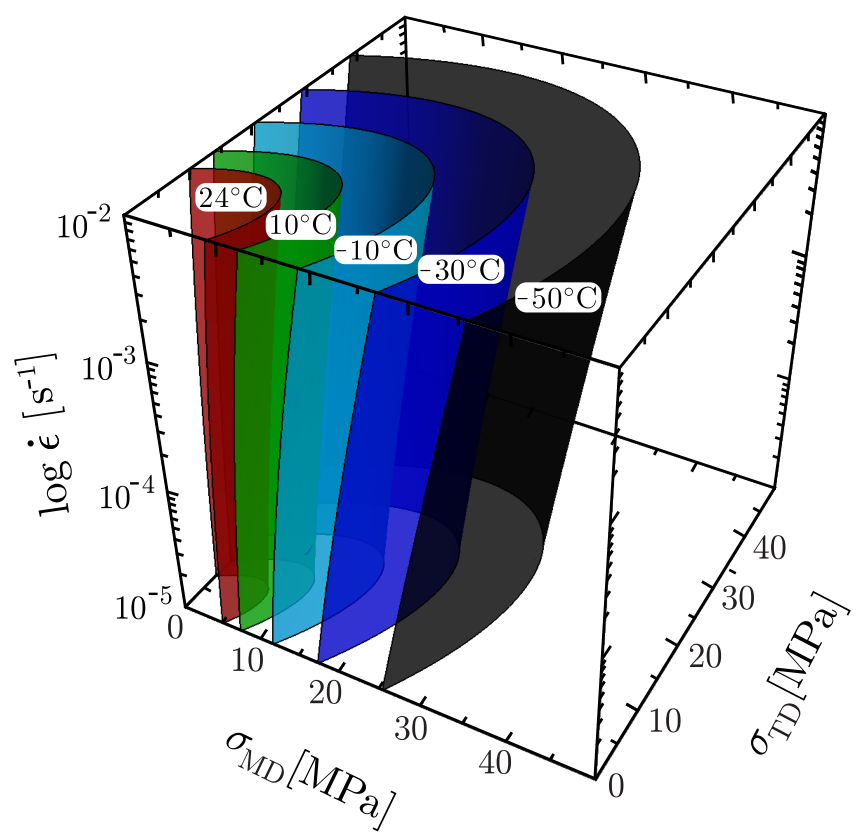

Figure 9: Three-dimensional representation of LLDPE film yield surfaces in principal stress coordinates as a function of the applied nominal deformation rate $\dot{\epsilon}$ for $T=[+24 ;+10 ;-10 ;-30 ;-50]^{\circ} \mathrm{C}$.

Substituting Eq. 22, into Eq. 21, the yield criterion can be finally written as

$$
\begin{aligned}
\left(\frac{\sigma_{\mathrm{MD}}}{\sigma_{y, \mathrm{MD}}}\right)^{2}+ & \left(\frac{\sigma_{\mathrm{TD}}}{\sigma_{y, \mathrm{TD}}}\right)^{2} \\
& -\left(\frac{1}{\left(\sigma_{y, \mathrm{MD}}\right)^{2}}+\frac{1}{\left(\sigma_{y, \mathrm{TD}}\right)^{2}}-\frac{1}{\left(\sigma_{y, b}\right)^{2}}\right) \sigma_{\mathrm{MD}} \sigma_{\mathrm{TD}}=1
\end{aligned}
$$

A graphical representation of the proposed criterion is presented in Fig. 9, where the elastoplastic limit is shown as a function of the deformation rate for five different temperatures, namely $T=[+24 ;+10 ;-10 ;-30 ;-50]^{\circ} \mathrm{C}$. Because only tensile stresses can be applied to a thin film, the yield surface has been defined only in the first quadrant of the $\sigma_{\mathrm{MD}}-\sigma_{\mathrm{TD}}$ plane. It can be noticed that the yield locus non-linearly expands with an increase of the strain rate and a decrease of the temperature. The yield loci shown in Fig. 9 can be drawn for lower or higher deformation rates and different temperatures in the rubbery state of the material. Finally, four horizontal cuts of the yield loci of Fig. 9 at $\dot{\epsilon}=[1 ; 0.1 ; 0.01 ; 0.001] \% \mathrm{~s}^{-1}$ are reported in Fig. 10 and compared with the experimentally measured yield stresses, showing an excellent agreement and confirming the accuracy of the developed criterion.

The experiments performed to develop the proposed yield criterion were carried out at uniform engineering strain rate. However, during the flight of a stratospheric balloon, the material experiences a biaxial stress state with different strain rates along the two principal directions. In this case, the evaluation of the margin from the inelastic region by means of Eq. 23) requires 

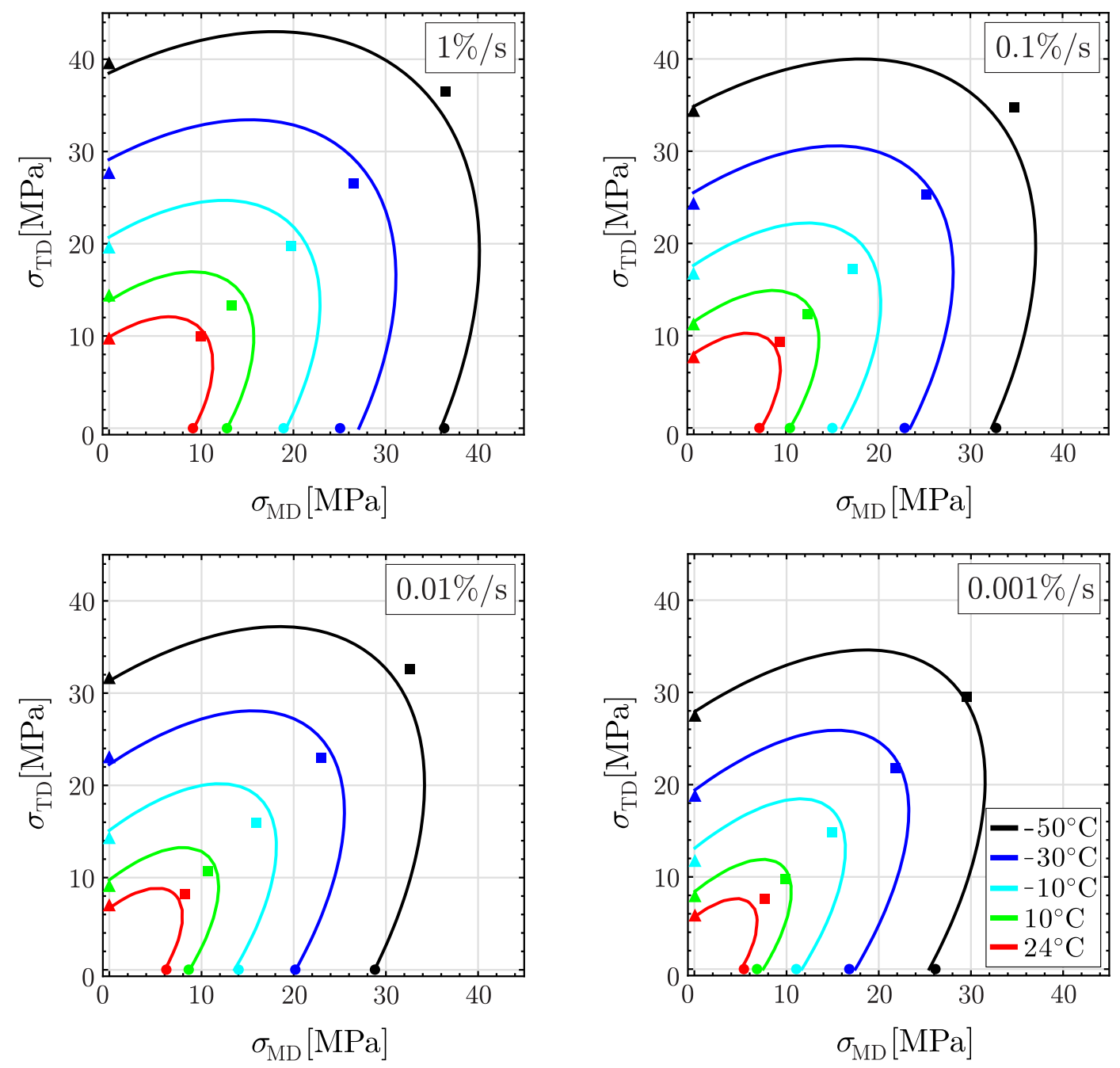

Figure 10: Yield loci for polyethylene thin film in principal stresses at different strain rates, namely $\dot{\epsilon}=[1 ; 0.1 ; 0.01 ; 0.001] \% \mathrm{~s}^{-1}$. Each plot shows the predictions of the proposed yield criterion together with the experimentally derived yield stresses for uniaxial tension along MD (circle), TD (triangle), and under equibiaxial tension (square) at $T=[+24 ;+10 ;-10 ;-30 ;-50]^{\circ} \mathrm{C}$.

as an input the strain rate values in the two principal directions, $\dot{\epsilon}_{\mathrm{MD}}$ and $\dot{\epsilon}_{\mathrm{TD}}$. The two different strain rates will define the yield stress along MD and TD by means of the cooperative equations, $\sigma_{y, \mathrm{MD}}\left(\dot{\epsilon}_{\mathrm{MD}}, T\right)$ and $\sigma_{y, \mathrm{TD}}\left(\dot{\epsilon}_{\mathrm{TD}}, T\right)$. Furthermore, the strain rate in an equibiaxial loading condition has to be selected in order to define $\sigma_{y, b}\left(\dot{\epsilon}_{b}, T\right)$. A reasonable assumption is to consider $\dot{\epsilon}_{b}=\left(\dot{\epsilon}_{\mathrm{MD}}+\dot{\epsilon}_{\mathrm{TD}}\right) / 2$, while $\dot{\epsilon}_{b}=\min \left[\dot{\epsilon}_{\mathrm{MD}} ; \dot{\epsilon}_{\mathrm{TD}}\right]$ might be adopted to obtain safer loci.

\section{Conclusions}

A yield criterion for rubbery semi-crystalline thin films has been developed by extending to anisotropic materials the cooperative model of yielding, a molecular formulation that describes the temperature and deformation rate dependence of the yield stress through the strain rate-temperature superposition principle. In the identification procedure of the model constants for different mechanical loadings, we have proposed to consider the activation volume $V$, the pre-exponential factor $\dot{\epsilon}_{0}$ and the activation energy $\Delta H$ as material parameters. The constants which define the anisotropy and loading conditions dependence of the yield stress are the initial internal stresses factors $\sigma_{i}(0)$ and $m$, as well as the number $n$ of polymer chain segments involved in the irreversible deformation process.

The presented molecular formulation of the viscoelastoplatic limit has been applied to the determination of the yield loci of a linear low density polyethylene film, namely StratoFilm 420. A detailed experimental investigation has been conducted in order to quantify the yield point via a strain recovery method. The material orthotropy has been studied through uniaxial tension tests along the two principal directions, while the equibiaxial threshold has been addressed by means of diaphragm inflation experiments performed over four order of magnitude of strain rates, $\dot{\epsilon}=[1 ; 0.1 ; 0.01 ; 0.001] \% \mathrm{~s}^{-1}$, and at five different thermal conditions above the glass transition temperature, namely $T=$ $[+24 ;+10 ;-10 ;-30 ;-50]^{\circ} \mathrm{C}$. As an alternative to the recovery method, it has been found that the $3 \%$ offset method provides 
accurate and fast determination of the uniaxial yield stresses for LLDPE films.

The experimental yield points of the material for the three investigated loading conditions have been modeled by means of the proposed formulation of the cooperative model, showing excellent agreement. An orthotropic plane stress criterion has been established to capture the evolution of the yield surface with temperature, deformation rate effects, and mechanical loading conditions, showing very good predictions of the experimental measurements.

The presented mathematical formulation and experimental campaign can be employed in the determination of the timedependent elastoplastic limits for any elastomeric membrane. The correct definition of the elastic domain is fundamental for the successful development of a viscoplastic characterization of the material response.

Acknowledgments We thank Prof. W.G. Knauss (Caltech), Dr. David Wakefield (Tensys Limited), Dr. James Rand (Winzen Engineering) and Prof. S. Ahzi (HBKU) for helpful discussions and comments. Financial support from the NASA Balloon Program Office is gratefully acknowledged.

\section{References}

[1] B. Lee, D. Parks, S. Ahzi, Micromechanical modeling of large plastic deformation and texture evolution in semi-crystalline polymers, J. mech. Phys. Solids 41 (1993) 1651-1687.

[2] J. van Dommelin, D. Parks, M. Boyce, W. Brekelmans, F. Baaijens, Micromechanical modeling of elasto-viscoplastic behavior of semi-crystalline polymers, J. mech. Phys. Solids 41 (2003) 1651-1687.

[3] B. Lee, A. Argon, D. Parks, S. Ahzi, Z. Bartczak, Simulation of large strain plastic deformation and texture evolution in high density polyethylene, Polymer 34 (17) (1993) 3555-3575.

[4] R. Meyer, L. Pruitt, The effect of cyclic true strain on the morphology, structure, and relaxation behavior of ultra high molecular weight polyethylene, Polymer 42 (12) (2001) 5293-5306.

[5] W. Cook, A. E. Mayr, G. H. Edward, Yielding behaviour in model epoxy thermosets - ii. temperature dependence, Polymer 39 (16) (1998) 37253733.

[6] H. Eyring, Viscosity, plasticity, and diffusion as examples of absolute reaction rates, J. Chem. Phys. 4 (1936) 283-291.

[7] T. Ree, H. Eyring, Theory for non-newtonian flow. i. solid plastic system, J. Appl. Phys. 26 (1955) 793.

[8] J. Roetling, Yield stress behaviour of poly(ethyl methacrylate) in the glass transition region, Polymer 6 (1965) 615-619.

[9] I. Ward, M. Wilding, Creep behavior of ultrahigh-modulus polyethylene: influence of draw ratio and polymer composition, J. Polym. Sci. 22 (4) (1984) 561-575.

[10] C. Bauwens-Crowet, J. Bauwens, G. Homes, Tensile yield-stress behavior of poly(vinyl chloride) and polycarbonate in the glass transition region, J. Polym. Sci. 7 (10) (1969) 1745-1754.

[11] A. Mulliken, M. Boyce, Mechanics of the rate-dependent elast-plastic deformation of glassy polymers from low to high strain rates, Int. J. Solids Struct. 43 (2006) 1331-1356.

[12] F. Povolo, E. B. Hermida, Phenomenological description of strain rate and temperature-dependent yield stress of pmma, J. Appl. Polym. Sci. 58 (1995) 55-68.

[13] N. W. J. Brooks, R. A. Duckett, I. M. Ward, Modeling of double yield points in polyethylene: temperature and strain rate dependence, J. Rheol. 39 (1995) 425-436.

[14] O. Gueguen, J. Richeton, S. Ahzi, A. Makradi, Micromechanically based formulation of the cooperative model for the yield bahavior of semicrystalline polymers, Acta Mater. 56 (2008) 1650-1655.
[15] D. Fotheringham, B. Cherrt, The role of recovery forces in the deformation of linear polyethylene, J. Mater. Sci. 13 (1978) 951-964.

[16] C. Bauwens-Crowet, J. Bauwens, G. Homes, Tensile yield-stress behavior of glassy polymers, J. Polym. Sci. 7 (1969) 735-742.

[17] R. E. Robertson, Theory for the plasticity of glassy polymers, J. Chem. Phys. 44 (1966) 3950-3956.

[18] A. Argon, A theory for the low-temperature plastic deformation of glassy polymers 28 (1973) 839-865.

[19] P. Bowden, S. Raha, A molecular model for yield and flow in amorphous glassy polymers making use of a dislocation analogue, Phil. Mag. 29 (1974) 149-166.

[20] E. Ghorbel, A viscoplastic constitutive model for polymeric materials, Int. J. Plasticity 24 (2008) 2032-2058.

[21] J. Rose, A. Duckett, I. Ward, The yield behaviour of poly(phenylenebibenzimidazole), J. Mater. Sci. 30 (1995) 5328-5334.

[22] I. Ward, Review: the yield behavior of polymers, J. Mater. Sci. 6 (1971) 1397-1417.

[23] D. Drucker, W. Prager, Soil mechanics and plastic analysis for limit design, Q. Appl. Math. 10 (2) (1951) 157-165.

[24] R. Raghava, R. Caddell, G. Yeh, The macroscopic yield behavior of polymers, J. Mater. Sci. 8 (1973) 225-232.

[25] J. Rottler, M. O. Robbins, Yield conditions for deformation of amorphous polymer glasses, Phys. Rev. E 64 (2001) 051801.

[26] V. Deshpande, N. Fleck, Multi-axial yield behaviour of polymer foams, Acta Mater. 49 (2001) 1859-1866.

[27] J. Richeton, S. Ahzi, L. Daridon, Y. Rmond, A formulation of the cooperative model for the yield stress of amorphous polymers for a wide ranfe of strain rates and temperatures, Polymer 46 (2005) 6035-6043.

[28] R. Hill, The mathematical theory of plasticity, Oxford University Press, 1950.

[29] R. N. Haward, G. Thackray, The use of a mathematical model to describe isothermal stress-strain curves in glassy thermoplastic, Proc. R. Soc. London A 302 (1968) 453-452.

[30] F. Povolo, G. Schwartz, E. B. Hermida, Temperature and strain rate dependence of the tensile yield stress of pvc, J. Appl. Polym. Sci. 61 (1996) 109-117.

[31] M. Bonner, R. A. Duckett, I. M. Ward, The creep behaviour of isotropic polyethylene, J. Mater. Sci. 34 (1999) 1885-1897.

[32] J. Richeton, S. Ahzi, K. S. Vecchio, F. C. Jiang, R. R. Adharapurapu, Influence of temperature and strain rate on the mechanical behavior of three amorphous polymers: Characterization and modeling of the compressive yield stress, Int. J. Solids Struct. 43 (2006) 2318-2335.

[33] R. Hill, A theory of the yielding and plastic flow of anisotropic metals, Proc. R. Soc. London A 193 (1947) 291-297.

[34] H. J. Cathey, The nasa super pressure balloon - a path to flight, Adv. Space Res. 44 (2009) 23-38.

[35] J. Rand, W. Sterling, A constitutive equation for stratospheric ballon materials, Adv. Space Res. 37 (2006) 2087-2091.

[36] J. Rand, D. Wakefield, Studies of thin film nonlinear viscoelasticity for superpressure balloons, Adv. Space Res. 45 (2010) 56-60.

[37] J. Li, K. Kwok, S. Pellegrino, Thermoviscoelastic models for polyethylene thin films, Mech. Time-Depend. Mat. 13 (1) (2016) 13-43.

[38] F. Bosi, S. Pellegrino, Nonlinear thermomechanical response and constitutive modeling of viscoelastic polyethylene membranes, Submitted.

[39] M. Sutton, J. Orteu, H. Schreier, Image correlation for shape, motion and deformation measurements: basic concepts, theory and applications, Springer, 2009.

[40] P. Reu, Introduction to digital image correlation: best practices and applications, Exp. Techniques 36 (1) (2012) 3-4.

[41] P. Reu, Calibration: strereo-calibration, Exp. Techniques 38 (1) (2014) $1-2$.

[42] I. Ward, J. Sweeney, Mechanical Properties of Solid Polymers, John Wiley and Sons Ltd, 2004, 2nd edition.

[43] R. W. Truss, P. L. Clarke, R. A. Duckett, I. M. Ward, The dependence of yield bahavior on temperature, pressure, and strain rate for linear polyethylenes of different molecular weight and morphology, Polym Phys. Ed. 22 (1984) 191-209.

[44] R. Quinson, J. Perez, M. Rink, A. Pavan, Yield criteria for amorphous glassy polymers, J. Mater. Sci. 32 (1997) 1371-1379.

[45] C. Marano, M. Rink, Shear yielding threshold and viscoelasticity in an amorphous glassy polymer: a study on a styrene-acrylonitrile copolymer, 
Polymer 42 (2001) 2113-2119. 